\title{
SOME INEQUALITIES ON POWER BOUNDED OPERATORS ACTING ON LOCALLY CONVEX SPACES
}

\author{
FLAVIUS PATER
}

Abstract. In this paper are presented some evaluations regarding characterizations of power boundedness of an operator from $\mathscr{B}_{\mathscr{P}}(X)$ algebra. This represents a generalization from the Banach spaces framework to the locally convex spaces where the operators acting on them are universally bounded. The authors extend some Ritt type theorems according to Kreiss conditions in the context of $\mathscr{B}_{\mathscr{P}}(X)$ algebra.

Mathematics subject classification (2010): 47A63, 47A10, 46A32.

Keywords and phrases: Convex algebra, power boundedness, locally bounded, operatorial inequalities.

\section{REFERENCES}

[1] G. R. Allan, A Spectral Theory for Locally Convex Algebras, Proc. Lon. Math. Soc. 15, 1965, 399 412.

[2] F. G. Bonales, R. V. Mendoza, Extending the formula to calculate the spectral radius of an operator, Proc. Amer. Math. Soc, 126 (1), 1998, 97-103.

[3] A. Chilana, Invariant subspaces for linear operators on locally convex spaces, J. London. Math. Soc, 2 (1970) , 493-503.

[4] I. Colojonara, Elemente de teorie spectrală, Editura Academiei Republicii Socialiste România, Bucureşti, 1968.

[5] N. Dunford, J. T. SchwartZ, Linear operators, part I, Interscience, New York, 1964.

[6] G. A. Joseph, Boundedness and Completeness in Locally Convex Spaces and Algebras, J. Austral. Math. Soc. 24 (Series A), 1977, 50-63.

[7] L. D. LemLe, L. M. Wu, Uniqueness of $C_{0}$-semigroups on a general locally convex vector space and an application, Semigroup Forum, 82 (2011), 485-496.

[8] L. D. Lemle, L. M. Wu, Unicité des pré-générateurs dans les espaces localement convexes, C. R. Acad. Sci. Paris, Ser. 1, 347 (2009), 1281-1284.

[9] L. D. LemLe, On Some Approximation Theorems for Power q-Bounded Operators on Locally Convex Vector Spaces, The Scientific World Journal 2014 (2014), 1-5.

[10] A. Michael, Locally Multiplicatively Convex Topological Algebras, Mem. Amer. Math. Soc., 11, 1952.

[11] R. T. Moore, Adjoints, numerical ranges and spectra of operators on locally convex spaces, Bull. Am. Math. Soc. 75 1969, p. 85-90.

[12] R. T. Moore, Banach Algebras of Operators on Locally Convex Spaces, Bull. Am. Math. Soc. 75, 1969, 68-73.

[13] B. NAGY, J. ZemaneK, A resolvent condition implying power boundedness, Studia Math. 134 (2), 1999, 143-151.

[14] O. Nevanlinna, Convergence of Iterations for Linear Equations, Birkhäuser, Basel, 1993.

[15] F. PATER, T. BÎNZAR, On some ergodic theorems for a universally bounded operator, Carpathian Journal of Mathematics, 26 (2010), 1, 97-102.

[16] F. Pater, L. D. Lemle, Y. Jiang and S. Serban, On Some Spectral Properties for Locally Bounded Operators, International Conference on Numerical Analysis and Applied Mathematics 2011, 1389, 524-527. 
[17] A. PAZY, Semigroups of Linear Operators and Applications to Partial Differential Equations, Springer-Verlag, New York, 1991.

[18] P. Perez-Carreras, J. Bonet, Barrelled locally convex spaces, North-Holland Math. Studies, North-Holland, Amsterdam, 1978.

[19] R. K. RitT, A condition that $\lim _{n \rightarrow \infty} n^{-1} T^{n}=0$, Proc. Amer. Math. Soc. 4, 1953, 898-899.

[20] A. L. Shields, On Möbius bounded operators, Acta Sci. Math., Szeged, 40, 1978, 371-374.

[21] L. Waelbroeck, Étude spectrale des algèbres complètes, Acad. Roy. Belg. Cl. Sci. Mem. Coll. in $8 \mathrm{deg}$ (2) 31, 1960 no. 7, 142 p. 